\title{
ON THE LEVY-PROKHOROV DISTANCE BETWEEN COUNTING PROCESSES
}

\author{
M. Nikunen and E. Valkeila
}

Let $(N, F)$ be a counting process with a deterministic compensator $A$ and let $(M, G)$ be another counting process. Suppose that $B$ is the compensator of $(M, G)$. Let us consider the restrictions of the processes to the interval $[0, T]$ and denote by $L P(M, N)$ the Levy-Prokhorov distance between the distributions of $M$ and $N$ on the Skorokhod space $D[0, T]$ (for the definition of the LevyProkhorov distance see for example [6]). We wish to find an upper bound for $L P(M, N)$.

We have in [5] derived an upper bound for $L P(M, N)$ in the case where the function $B$ is continuous. Let us now assume that $A_{t}=t$ for all $t \geq 0$, i.e., $N$ is a standard Poisson process and the compensator $B$ is deterministic and continuous. Then our result from [5] gives

$$
L P(M, N) \leq \sup _{t \leq T}\left|B_{t}-t\right|+\left|B_{T}-T\right| .
$$

In the present note we extend this result to a more general class of compensators. Before stating our main theorem we note that, in what follows, we define $\Delta X_{t}=$ $X_{t}-X_{t-}$ for a cadlag-process $X$.

Theorem. Suppose that $N$ is a standard Poisson process and the compensator $B$ is deterministic (but not necessarily continuous). Then

$$
L P(M, N) \leq \sup _{t \leq T}\left|B_{t}-t\right|+\left|B_{T}-T\right|+\frac{3}{2} \sum_{t \leq T}\left(\Delta B_{t}\right)^{2}+\Delta B_{T} .
$$

Before the proof we discuss the upper bound in (2). Let $X_{1}, \ldots, X_{n}$ be independent Bernoulli random variables with $P\left\{X_{i}=1\right\}=1 / n, i=1, \ldots, n$. If

$$
M_{t}=\sum_{i=1}^{[n t]} X_{i}
$$

then $M$ is a counting process with compensator $B, B_{t}=[n t] / n$. Let $T=1$. From (2) we get the result of Dudley [2] (see also Whitt [6])

$$
L P(M, N) \leq \frac{7}{2 n}
$$


Dudley shows in [2] that this bound cannot be improved to order $o\left(n^{-1}\right)$.

To continue our discussion about (2) we recall the following special case of results in Kabanov et al. [3]:

If $\left(M^{n}\right)$ is a sequence of counting processes with deterministic compensators $B^{n}$ such that

$$
B_{t}^{n} \longrightarrow t
$$

for every $t \geq 0$, then

$$
M^{n} \stackrel{L(D)}{\longrightarrow} N, \text { as } n \rightarrow \infty
$$

(here $\stackrel{L(D)}{\longrightarrow}$ means weak convergence in the Skorokhod space $D[0, T])$. It is easy to check that if (3) holds the upper bound given by (2) tends to zero as $n \rightarrow \infty$. Kabanov et al. [3] show that (3) is a necessary condition for the above weak convergence.

Proof of the Theorem. Let $\left\{0=t_{0}, \ldots, t_{n}=T\right\}$ be a partition of the interval $[0, T]$ such that $t_{i}=i T / n, i=0, \ldots, n$. If $X$ is a process, then we write $f^{n}(X)$ for the discretized process

$$
f_{t}^{n}(X)=X_{t_{i}}, \quad \text { if } t_{i} \leq t<t_{i+1},
$$

$i=0, \ldots, n-1, f_{T}^{n}(X)=X_{T}$. If $X$ is a cadlag-process, then it is not difficult to see that $f^{n}(X)$ converges weakly to $X$ on $D[0, T]$ as $n \rightarrow \infty$ so that $L P\left(f^{n}(X), X\right) \longrightarrow 0$ as $n \rightarrow \infty$.

Let $g$ be a continuous nondecreasing function such that $g\left(t_{i}\right)=B_{t_{i}}, i=$ $0, \ldots, n$ and denote by $H$ the counting process $H_{t}=N_{g(t)}$. Then $g$ is the compensator of the process $H$ (with respect to the natural $\sigma$-field). Now we can estimate $L P(M, N)$ in the following way:

$$
L P(M, N) \leq L P\left(M, f^{n}(M)\right)+L P\left(f^{n}(M), f^{n}(H)\right)+L P\left(f^{n}(H), H\right)+L P(H, N) .
$$

As noted above, $\operatorname{LP}\left(M, f^{n}(M)\right) \longrightarrow 0$ as $n \rightarrow \infty$.

Before giving an upper bound for the term $L P\left(f^{n}(M), f^{n}(H)\right)$ we need some notation. Put $\Delta_{i}^{n}(B)=B_{t_{i}}-B_{t_{i-1}}$ for $i=1, \ldots, n$. Denote by $V(M, H)$ the variation distance between the distributions of $M$ and $H$ on $D[0, T]$ and by $V^{n}(M, H)$ the variation distance between the distributions of $\left(M_{t_{0}}, \ldots, M_{t_{n}}\right)$ and $\left(H_{t_{0}}, \ldots, H_{t_{n}}\right)$. Note that $V\left(f^{n}(M), f^{n}(H)\right)=V^{n}(M, H)$. Hence we have also $L P\left(f^{n}(M), f^{n}(H)\right) \leq V^{n}(M, H)$. According to a result of Brown [1] or Kabanov et. al [4]

$$
V^{n}(M, H) \leq \sum_{i=1}^{n}\left|\Delta_{i}^{n}(B)-\Delta_{i}^{n}(g)\right|+\sum_{t \leq T}\left(\Delta B_{t}\right)^{2}
$$


But here $g\left(t_{i}\right)=B_{t_{i}}$ and so $\left|\Delta_{i}^{n}(B)-\Delta_{i}^{n}(g)\right|=0$ in (4). Hence we have for the term $L P\left(f^{n}(M), f^{n}(H)\right)$ the following upper bound:

$$
L P\left(f^{n}(M), f^{n}(H)\right) \leq \sum_{t \leq T}\left(\Delta B_{t}\right)^{2} .
$$

Note that $H$ is a counting process with continuous compensator $g$. It is easily seen that

$$
\sup _{t \leq T}|g(t)-t| \leq \sup _{t \leq T}\left|B_{t}-t\right|+\frac{T}{n} .
$$

This and (1) yield

$$
L P(H, N) \leq \sup _{t \leq T}\left|B_{t}-t\right|+\frac{T}{n}+\left|B_{T}-T\right| .
$$

Next we derive an upper bound for $L P\left(f^{n}(H), H\right)$. We use the method of Dudley [2] (see also Whitt [6]). Denote by $d_{T}$ the Skorokhod distance on $D[0, T]$. For any $\delta>0$ we have

$$
L P\left(f^{n}(H), H\right) \leq \max \left\{\delta, P\left\{d_{T}\left(f^{n}(H), H\right) \geq \delta\right\}\right\} .
$$

Define

$$
F=\left\{H_{T}-H_{t_{n-1}} \geq 1\right\} \quad \text { and } G=\bigcup_{i=1}^{n}\left\{H_{t_{i}}-H_{t_{i-1}} \geq 2\right\} .
$$

Put $C=F \cup G$. Dudley shows in [2] that on the complement of the set $C$ we have $d_{T}\left(f^{n}(H), H\right) \leq T / n$. First we note the following inequality:

$P\left(d_{T}\left(f^{n}(H), H\right) \geq \delta\right) \leq P\left(\left\{d_{T}\left(f^{n}(H), H\right) \geq \delta\right\} \cap C\right)+P\left(\left\{d_{T}\left(f^{n}(H), H\right) \geq \delta\right\} \cap C^{c}\right)$

so that

$$
L P\left(f^{n}(H), H\right) \leq P(F)+P(G)+\frac{T}{n} .
$$

Because $g\left(t_{i}\right)=B_{t_{i}}$, we have in (7):

$$
P(F) \leq B_{T}-B_{t_{n-1}} \text { and } P(G) \leq \frac{1}{2} \sum_{i=1}^{n}\left(\Delta_{i}^{n}(B)\right)^{2} .
$$

Letting now $n \rightarrow \infty$ in (7) we get

$$
\underset{n}{\limsup } L P\left(f^{n}(H), H\right) \leq \Delta B_{T}+\frac{1}{2} \sum_{t \leq T}\left(\Delta B_{t}\right)^{2} .
$$

The claim (2) follows now from (5), (6) and (8), by letting $n \rightarrow \infty$. This completes our proof. 


\section{References}

[1] Brown, T.C.: Some Poisson approximations using compensators. - Ann. Probab. 11, 1983, 726-744.

[2] Dudley, R.M.: Speeds of metric probability convergence, - Z. Wahrsch. Verw. Gebiete $22,1972,323-332$.

[3] Kabanov, YU.M., R.Sh. Liptser, and A.N. ShiRYayev: Some limit theorems for simple point processes (a martingale approach). - Stochastics 3, 1980, 203-216.

[4] Kabanov, Yu.M., R.Sh. Liptser, and A.N. Shiryayev: Weak and strong convergence of distributions of counting processes. - Theor. Probability Appl. 28, 1983, 303-335 (English translation of Teor. Veroyatnost. i Primenen. 28, 2, 1983, 288-319).

[5] Nikunen M., and E. Valkeila : Metric distances between counting processes. - In Statistics and control of random processes, Steklov seminar, 1984, edited by N.V. Krylov, R.Sh. Liptser and A.A. Novikov, Optimization Software Inc., New York, 1985, 377388.

[6] Whiтt, W.: On the quality of Poisson approximations. - Z. Wahrsch. Verw. Gebiete 28, $1973,23-26$.

University of Helsinki

Department of Mathematics

Hallituskatu 15

SF-00100 Helsinki

Finland
University of Helsinki

Computing Centre

Teollisuuskatu 23

SF-00510 Helsinki

Finland 